\title{
ATENÇÃO DOMICILIAR EM SAÚDE NO BRASIL: VISÃO DESSA POLÍTICA POR UMA REVISÃO INTEGRATIVA
}

\section{Home health care in Brazil: a glance at this policy through an integrative review \\ Atención domiciliaria en salud de Brasil: opinión sobre esa política a través de una revisión integrativa}

\author{
Carmem Rita Sampaio de Sousa iD \\ Universidade Federal do Ceará - UFC - Fortaleza (CE) - Brasil \\ Maria do Socorro de Sousa (iD \\ Universidade Estadual do Ceará - UECE - Fortaleza (CE) - Brasil
}

\section{RESUMO}

Objetivo: Revisar a produção científica acerca da política da atenção domiciliar em saúde no Brasil. Métodos: Revisão integrativa de literatura realizada no período de agosto a outubro de 2018, nas seguintes bases de dados: Medical Literature Analysis and Retrieval System Online (MEDLINE), consultada por meio do PubMed; Literatura Latino-Americana e do Caribe em Ciências da Saúde (LILACS), consultados pela Biblioteca Virtual em Saúde (BVS) e SciELO (Scientific Eletronic Library Online). Foram utilizados, para busca dos artigos, os seguintes descritores e suas combinações nas línguas portuguesa, inglesa e espanhola: "atenção domiciliar", "assistência domiciliar", "serviços hospitalares de assistência domiciliar". Selecionaram-se 20 artigos na análise final. Resultados: Os achados foram categorizados na seguinte forma: atenção domiciliar como subsídio do sistema público de saúde e atenção domiciliar na suplementação à saúde, dada a importância da atenção domiciliar integrada à rede de atenção à saúde. Conclusão: As evidências científicas apontam a atenção domiciliar como uma ferramenta a ser utilizada na reorganização do sistema de saúde, prevenção de agravos e promoção da saúde.

Descritores: Assistência Domiciliar; Serviços Hospitalares de Assistência Domiciliar; Políticas Públicas de Saúde.

\section{ABSTRACT}

Objective: To review the scientific production about home care in Brazil. Methods: An integrative review of the literature was conducted from August to October 2018 on the following databases: Medical Literature Analysis and Retrieval System Online (MEDLINE), accessed via PubMed; Latin American and Caribbean Health Sciences Literature (LILACS), accessed via the Virtual Health Library (VHL), and SciELO (Scientific Electronic Library Online). The following descriptors and their combinations in Portuguese, English and Spanish were used to search for articles: "Home Care", "Home Nursing", and "Hospital-based Home Care Services". A total of 20 articles were selected for the final analysis. Results: The findings were categorized as Home Care as an aid to the public health system and Home care in complement health care, given the importance of home care integrated into the health care network. Conclusion: Scientific evidence points to home care as a tool to be used in the reorganization of the health system, in disease prevention, and in health promotion.

Descriptors: Home Care; Hospital-based Home Care Services; Public Health Policies.

\section{RESUMEN}

Objetivo: Revisar la producción científica sobre la política de la atención domiciliaria de salud en Brasil. Métodos: Revisión integrativa de la literatura realizada en el período entre agosto y octubre de 2018 en las bases de datos Medical Literature Analysis and Retrieval System Online (MEDLINE), consultada a través de PubMed; Literatura Latino-Americana y del Caribe en las Ciencias de la Salud (LILACS) consultada a través de la Biblioteca Virtual en Salud (BVS) y SciELO (Scientific Eletronic Library Online). Se ha utilizado los descriptores y sus combinaciones en los idiomas portugués, inglés y español a continuación: "atención domiciliaria", "asistencia domiciliaria", "servicios hospitalarios de asistencia domiciliaria". Fueron elegidos 20 artículos

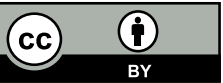


para el análisis final. Resultados: Se han categorizado los hallazgos en: atención domiciliaria como subsidio del sistema público de salud y atención domiciliaria para la suplementación de salud debido la importancia de la atención domiciliaria integrada a la red de atención de salud. Conclusión: Las evidencias científicas señalan la atención domiciliaria como una herramienta para utilizarse en la reorganización del sistema de salud, la prevención de agravios y la promoción de la salud.

Descriptores: Atención Domiciliaria de Salud; Servicios de Atención a Domicilio Provisto por Hospital; Políticas Públicas de Salud.

\section{INTRODUÇÃO}

A população brasileira vem vivenciando tempos de crises econômicas, políticas e sociais, o que elucida a importância dos serviços de saúde pública para encontrar alternativas para a melhoria da assistência ao paciente crônico(1).

O envelhecimento populacional e o aumento da sobrevida das situações de cronicidade nas diversas faixas etárias exigem do Sistema Único de Saúde (SUS) a oferta e o aporte de recursos necessários para uma assistência de qualidade ${ }^{(2)}$.

Diante disso, a atenção domiciliar vem ganhando destaque como alternativa para a continuidade da assistência ao usuário, garantindo os princípios básicos do SUS, como a integralidade e a equidade, mas com a necessidade de gestão, acompanhamento e financiamento para garantir o objetivo que se propõe a alcançar na qualidade de vida da população $0^{(3,4)}$.

A atenção domiciliar é definida pelo termo genérico que envolve ações de promoção à saúde, prevenção, tratamento de doenças e reabilitação desenvolvidas em domicílio, tendo como objetivo principal assegurar o integralidade do paciente em seu ambiente domiciliar ${ }^{(5)}$.

Em 2016, foi aprovada a Portaria n. ${ }^{\circ} 825$, que redefine o Programa Melhor em Casa, e considera que a atenção domiciliar, modalidade de atenção à saúde integrada à Rede de Atenção à Saúde (RAS), é caracterizada por um conjunto de ações de prevenção e tratamento de doenças, reabilitação, paliação e promoção à saúde, prestadas em domicílio, o que garante a continuidade de cuidados $^{(6,7)}$.

Entendendo a necessidade de construir políticas saudáveis que possibilitem a saúde da população através de desenvolvimento de habilidades individuais e coletivas, promovendo a qualidade de vida e reduzindo a fragilidade e risco à saúde ${ }^{(8)}$, a atenção domiciliar vem se estabelecendo como uma ferramenta de continuidade da assistência através de ações de promoção da saúde e prevenção de agravos ${ }^{(9,10)}$.

Considerando a importância da promoção da saúde no contexto da atenção domiciliar buscou-se, neste artigo, revisar a produção científica acerca da política da atenção domiciliar em saúde no Brasil.

\section{MÉTODOS}

Optou-se por uma revisão integrativa da literatura, tendo como etapas: a elaboração da questão norteadora; a definição das bases de dados e o estabelecimento de critérios para inclusão e exclusão de estudos/amostragem ou busca na literatura; a definição das informações a serem extraídas dos estudos selecionados; a avaliação dos estudos incluídos na revisão integrativa; a interpretação dos resultados e a apresentação da revisão/síntese do conhecimento ${ }^{(11,12)}$.

Identificaram-se os artigos por meio de busca bibliográfica, realizada no período de agosto a outubro de 2018 , para responder à pergunta norteadora: Como a política de atenção à saúde está sendo avaliada pela comunidade cientifica? Utilizaram-se as seguintes bases de dados: Medical Literature Analysis and Retrieval System Online (MEDLINE), consultada por meio do PubMed; Literatura Latino-Americana e do Caribe em Ciências da Saúde (LILACS), consultados pela Biblioteca Virtual em Saude (BVS) e SciELO (Scientific Eletronic Library Online).

Utilizaram-se as combinações entre os descritores: "atenção domiciliar" AND "assistência domiciliar" AND "serviços hospitalares de assistência domiciliar", em suas representações em português e suas respectivas traduções para o inglês e espanhol. Todos os descritores foram definidos na plataforma dos Descritores em Ciências da Saúde (DeCS).

Excluíram-se os artigos que contemplavam a visita domiciliar realizada pela Estratégias de Saúde da Família (ESF), artigos de opinião, revisões bibliográficas, editorais, carta aos editores e comentários, além de artigos que retratassem a realidade da atenção domiciliar no exterior. 
Na busca inicial pelas associações dos descritores encontrou-se um total de 620 publicações. Através da leitura dos títulos e dos resumos foi possível excluir aquelas em duplicidade nas diferentes bases de dados, os estudos que não atendiam aos critérios de inclusão e/ou ao tema proposto. Dessa forma, foram selecionados 42 artigos para leitura na íntegra. Destes, 17 não foram localizados na íntegra e cinco rejeitados por não possuírem fascículo, ano ou volume. Dessa forma, dos 42 lidos na íntegra, 20 responderam à questão norteadora e, portanto, constituíram a amostra final desta revisão.

\section{RESULTADOS}

Foram selecionados 20 artigos que atendiam aos critérios de inclusão para alcance do objetivo proposto, todos publicados entre 2008 e 2018. O maior número de publicações incluídas ocorreu na base SciELO (85\%), seguida pela MEDLINE ( $8 \%$ ) e pela LILACS (7\%). Houve predomínio do idioma português (19 publicações) e de publicações em revistas especializadas da área de Enfermagem.

Os principais achados dos estudos evidenciam que a atenção domiciliar possui uma complexidade e diversidade de ações, o que permitiu a construção de dois eixos temáticos: 1. Atenção domiciliar como política pública de saúde; 2. Atenção domiciliar na suplementação à saúde.

\section{Atenção domiciliar como política pública de saúde}

Através da análise dos artigos, observou-se que a atenção domiciliar é uma estratégia política ainda em fase de implantação, mas importante na efetivação dos princípios do SUS, tais como integralidade, equidade, preservação da autonomia das pessoas e igualdade da assistência à saúde.

Os artigos selecionados trouxeram em seus discursos a integralidade como uma das principais características da atenção domiciliar. Um estudo qualitativo com o objetivo de analisar práticas de atenção domiciliar de serviços ambulatoriais e hospitalares e sua constituição como rede substitutiva de cuidado em saúde concluiu que, na essência da atenção domiciliar, é preciso que se considerem elementos como a integralidade do cuidado, a racionalidade econômico-financeira, os sujeitos do cuidado ${ }^{(4)}$ e a articulação com os demais serviços de saúde ${ }^{(13)}$.

Para a implantação efetiva da atenção domiciliar é imprescindível a integralidade do cuidado, garantindo continuidade da assistência, devendo ser ampliada no âmbito do sistema público de saúde, permitindo maior humanização ao paciente e a individualização da assistência prestada ${ }^{(13,14)}$. Se a integralidade do indivíduo não for priorizada na atenção domiciliar, pode-se colocar em risco a efetivação dessa modalidade de assistência. Constata-se essa colocação no estudo que analisou o processo de desospitalização em um hospital público geral de Minas Gerais na perspectiva dos diretores, dos profissionais e usuários, e resultou que, se houverem fragilidades no processo de desospitalização segura, a integralidade e a continuidade dos cuidados podem ser comprometidas na execução da assistência domiciliar(15).

A atenção domiciliar constitui importante estratégia para o alcance de práticas pautadas na integralidade em três sentidos: sentido vertical, que pressupõe a busca das necessidades dos usuários a partir de um olhar integral e ampliado; no sentido horizontal, que evidencia que as respostas às necessidades dos usuários geralmente não são obtidas por meio do primeiro ou único contato com o sistema de saúde, e no sentido transversal, que propicia a atenção voltada para condições que não são, por vezes, valorizadas no âmbito hospitalar ${ }^{(16)}$.

A equidade de assistência foi trazida em estudo realizado em 23 estados brasileiros e que objetivou identificar a prevalência do recebimento de atenção domiciliar entre os idosos brasileiros e sua associação com os fatores demográficos e socioeconômicos, as condições de saúde e a utilização de serviços de saúde. A ocorrência foi maior entre as mulheres, os mais velhos, com menor escolaridade e poder aquisitivo, com diagnóstico de morbidade crônica, história de queda, hospitalização prévia e consulta médica nos últimos três meses. Os resultados destacam a maior utilização da assistência domiciliar por idosos mais vulneráveis, o que indica uma contribuição da assistência domiciliar à promoção da equidade na atenção à saúde no país, principalmente em decorrência da expansão da ESF(17).

A associação entre a atenção domiciliar e a autonomia e humanização dos cuidados foi observada em alguns estudos $^{(1,18,19)}$. A atenção domiciliar tem como características: a desospitalização, favorecendo a rotatividade de leitos; a continuidade da assistência a ser prestada, reduzindo as internações hospitalares dos pacientes em acompanhamento e os custos por internações prolongadas; e a humanização de cuidados através do plano terapêutico e classificação desse cuidado, com autonomia das famílias. Ocorre em associação a um trabalho multidisciplinar e interdisciplinar, buscando a resolutividade no primeiro contato, prestando atenção contínua e longitudinal, com território definido, e promovendo as relações interpessoais (trabalhadores e usuários) para um cuidado humanizado ${ }^{(14)}$. 
A atenção domiciliar amplia a autonomia usuário/família, remontando à noção de família como unidade de organização social e operadora do cuidado imediato a partir da utilização do domicílio como um lugar terapêutico, caracterizando a substitutividade da organização da atenção(19,20).

Essa modalidade de atendimento, o domiciliar, vem se mostrando uma forma de cuidado que oferece ao paciente e a seus familiares a possibilidade de ter uma assistência individualizada, o que traz certa segurança aos familiares. Assim, faz-se necessária uma gestão do cuidado que vise à qualidade assistencial e melhores condições de saúde para os indivíduos ${ }^{(16)}$.

No entanto, ao avaliar o conceito da autonomia dos cuidados domiciliares a partir do olhar do cuidador, ressaltase a falta de apoio do setor saúde, o que sobrecarrega a família em relação ao cuidado. Muitos processos judiciais ocorrem devido ao desespero familiar com a falta de efetivo apoio aos cuidadores, pois não há serviços de apoio social domiciliar. Dessa forma, a atenção domiciliar é uma possibilidade de organização do serviço de saúde que colabora para preservar a autonomia da pessoa dependente de cuidado em longo prazo, que vivencia de perto a vulnerabilidade humana, o que indica a necessidade de associar serviços de saúde a serviços de apoio sociais ${ }^{(17,19,20)}$.

Por último, há a necessidade de institucionalizar uma rede de atenção à saúde dos usuários para a efetivação da atenção domiciliar como política pública de saúde, ponto discutido em alguns artigos. A rede de atenção à saúde tem papel determinante na gestão dos cuidados ampliados pelos seus serviços para a tomada de decisão da equipe sobre as prioridades e a melhor forma de atender às necessidades de saúde daqueles que precisam de cuidados domiciliares no território em saúde. No entanto há algumas dificuldades encontradas para a articulação do Programa de Atenção Domiciliar (PAD) com os outros serviços da rede, que ressaltam a importância dessa articulação por ser considerada significativa para se efetivar os princípios doutrinários do SUS ${ }^{(18,21,22)}$.

Complementando a visão dos estudos citados acima, deve-se esclarecer a importância da atenção domiciliar integrada à rede de atenção à saúde, potencializando a transversalidade da atenção ao colocar o usuário e suas necessidades no centro, e, para isso, o Serviço de Atenção Domiciliar (SAD) deve ter estabelecidos e devem ser pactuados fluxos e protocolos com o conjunto dos pontos de atenção dessa rede ${ }^{(18,22)}$.

\section{Atenção domiciliar na suplementação à saúde}

Ainda ressaltando a importância da atenção domiciliar como política pública de saúde, os artigos selecionados para comporem esta revisão trouxeram conceitos dessa modalidade assistencial dentro da suplementação da saúde, justificada pela visão de que a assistência domiciliar esteve sempre associada com serviços particulares de saúde, desde a sua origem, nos Estados Unidos.

A atenção domiciliar é um processo de reestruturação produtiva, isto é, há importantes mudanças no modo de produção do cuidado, e complementam que a atenção domiciliar na saúde suplementar não é regulamentada pela Agência Nacional de Saúde, pois obedece apenas à regulamentação da Anvisa. Ela não consta nos contratos das operadoras com sua clientela, sendo considerada um produto adicional ofertado aos beneficiários e, sendo assim, a definição da modelagem e do funcionamento dos programas fica a cargo da própria operadora e, quando for o caso, isto é feito conjuntamente com a prestadora desse tipo de serviço. Para isso, utiliza-se de trabalho em rede, com multidisciplinaridade do cuidado através de um projeto terapêutico integrado ${ }^{(23)}$.

Para demonstrar a atuação da atenção domiciliar dentro da estrutura dos serviços da saúde suplementar, ressalta-se um estudo que permitiu descrever e analisar a produção do cuidado no PAD de uma cooperativa médica, o qual observou que a atenção domiciliar estava sendo prestada com a internação domiciliar, na qual o beneficiário tem a assistência conforme o seu quadro clínico, visando à redução de custos da operadora referente às internações hospitalares. Nesses casos, de internação domiciliar, a operadora de saúde fornecia todos os materiais, equipamentos, insumos e profissionais ao cliente, tornando-se oneroso para a cooperativa em consequência da frequente judicialização da saúde e da transferência da responsabilidade da família para o plano de saúde ${ }^{(24)}$.

Entende-se por internação domiciliar o conjunto de atividades prestadas no domicílio a pessoas clinicamente estáveis, que podem ser assistidas em casa de forma integral e contínua, mas que requerem cuidados mais intensos, com oferta de tecnologias, recursos humanos, equipamentos, materiais e medicamentos ${ }^{(3)}$.

Estudo mostra que a atenção domiciliar foi indicada como a principal atividade incluída pelas operadoras no rol de promoção da saúde e transferida a sua execução para as prestadoras terceirizadas. Outro ponto importante é a racionalização de custos, sendo determinante na oferta dos programas de promoção da saúde e atenção domiciliar, mas o fazem com um discurso crítico que reconhece a necessidade de contenção de gastos, e não negam que há outros interesses da família, da operadora, do hospital, do beneficiário que conformam essa relação(23-25).

Ao analisar os PAD na saúde suplementar do ponto de vista do direito à saúde, avalia que a desregulamentação dessa atenção na saúde suplementar foi revelada como um desafio para a garantia do direito à saúde, prevalecendo 
a oferta segundo os interesses das operadoras, que adotam estratégias para evitar a judicialização, como não divulgar o benefício.

A suspensão da assistência por decisão unilateral da operadora e a transferência de responsabilidades para o sistema público de saúde e para as famílias afrontam o direito dos usuários de receberem serviços na modalidade que melhor respondam a determinadas situações de saúde. Conclui-se que a atenção domiciliar na saúde suplementar é permeada por tensões que evidenciam a urgência de maior regulamentação no campo ${ }^{(26,27)}$.

\section{DISCUSSÃO}

Para iniciar a discussão dos dados relevantes da presente pesquisa, optou-se em registrar a Lei n. ${ }^{\circ} 8080$, de 19 de setembro de 1990, que dispõe a Lei Orgânica de Saúde, que resolve sobre as condições para a promoção, proteção e recuperação da saúde, a organização e o funcionamento dos serviços correspondentes ${ }^{(2)}$.

Baseado nessas determinações, ressalta-se que os princípios que regem o sistema de saúde brasileiro, ou seja, a universalidade, a integralidade e a equidade, bem como as diretrizes que operacionalizam o referido sistema, como descentralização, hierarquização e participação comunitária, não têm sido suficientes para garantir a universalização da saúde em virtude de seu caráter dual, no qual a maior parte dos recursos públicos é direcionada aos serviços privados.

Persiste uma reprodução de dois mundos desiguais: o dos pobres, que recorrem somente ao SUS, e o dos que possuem planos de saúde ou pagam os serviços diretamente, mas que também são usuários do sistema público. Há hospitais e clínicas para todos os níveis: para os pobres que só dispõem do SUS; para pobres que pagam planos de saúde de baixo custo; para pessoas com renda média, que contratam planos ou seguros de saúde de custo maior; e para os ricos, que frequentam os maiores centros médicos de ponta ou vão se tratar nos países de primeiro mundo ${ }^{(18,26,27)}$.

Levando em consideração a atenção domiciliar e os princípios que regem o SUS, os estudos nos mostram que essa modalidade se constitui numa alternativa para tal fim ao assegurar a integralidade e a continuidade da assistência ${ }^{(25)}$ e ao objetivar a humanização do cuidado ${ }^{(17)}$, com autonomia do cuidado pelo próprio usuário, bem como pela família, em condições de impossibilidade de autocuidado( ${ }^{(18)}$.

A efetivação dessa atenção domiciliar foi descrita através Portaria n. ${ }^{\circ} 825$ de 2016, que redefine a atenção domiciliar considerandoa como incorporação tecnológica de caráter substitutivo ou complementar à intervenção hospitalar de baixa e média complexidade, aos cuidados iniciados nos Serviços de Atenção à Urgência e Emergência, e complementar à Atenção Básica ${ }^{(10-28)}$, sendo estruturada na perspectiva das Redes de Atenção à Saúde, através das linhas de cuidado por meio de práticas clínicas cuidadoras baseadas nas necessidades do usuário, reduzindo a fragmentação da assistência ${ }^{(24,26)}$.

Na suplementação da saúde, os beneficiários se sentem receosos pela assistência ofertada pelos planos de saúde e apontam que algumas das estratégias de redução de custos têm afetado a qualidade e afrontado a integralidade do cuidado. A sustentabilidade econômica do setor privado depende do uso da lógica biomédica e da fragmentação do cuidado, acabando por produzir uma demanda crescente por procedimentos e serviços cada vez mais especializados, de alta densidade tecnológica e alto custo, que, diante do aumento dos gastos na lógica de ampliação dos lucros, deve ser sempre reduzido, sendo redirecionados para os custos vindos do governo na maioria das vezes ${ }^{(27,28)}$.

Ainda no ano de 2013, o Ministério da Saúde lançou a Portaria n. ${ }^{\circ} 1208$, que dispõe sobre a integração do Programa Melhor em Casa (de atenção domiciliar no âmbito do SUS) com o Programa SOS Emergências, ambos inseridos na Rede de Atenção às Urgências. A integração da atenção domiciliar com o Programa SOS Emergências tem como objetivos: evitar internações hospitalares desnecessárias de pacientes atendidos nas Portas de Entrada Hospitalares de Urgência que compõem o Programa SOS Emergências; proporcionar o abreviamento de internações hospitalares, quando indicado clinicamente, possibilitando ampliação dos leitos de retaguarda para as Portas de Entrada Hospitalares de Urgência que compõem o Programa SOS Emergências; humanizar o cuidado aos pacientes em internação hospitalar e atendidos nas Portas de Entrada Hospitalares de Urgência que compõem o Programa SOS Emergências, possibilitando que sejam acompanhados no ambiente domiciliar pela Equipe Multiprofissional de Atenção Domiciliar (EMAD) ${ }^{(10,25,28)}$.

Segundo dados do Ministério da Saúde, o Programa Melhor em Casa abrange 135 municípios, com 433 equipes implantadas, e liberou cerca de 3,5 mil leitos hospitalares por dia para outros pacientes do SUS. Dos pacientes atualmente atendidos em domicílio por equipes do programa, quase $70 \%$ têm 60 anos ou mais, dos quais quase a metade (31\%) tem 80 anos ou mais. São portadores de doenças crônicas com dificuldade de locomoção até os 
serviços de saúde; usuários de sondas, traqueostomias ou dependentes de ventilação mecânica ou oxigenioterapia; pacientes em processo de reabilitação motora ou respiratória; os que precisam de curativos complexos, em pré ou pós-operatório; recém-nascidos com baixo peso, entre outros. $\mathrm{O}$ alívio que o programa representou para pacientes e seus familiares foi demonstrado em uma pesquisa de satisfação dos usuários, realizada pela ouvidoria do SUS, com mais de 2 mil pacientes ou cuidadores entrevistados. De acordo com o levantamento, 93,9\% dos entrevistados atribuíram nota entre 7 e 10 ao atendimento prestado pelas equipes, sendo que $70 \%$ deram nota 10 e $95,9 \%$ disseram que recomendariam o serviço a amigos e familiares ${ }^{(29)}$.

A efetividade do Programa Melhor em Casa foi encontrada em três artigos científicos nas bases de dados pesquisadas. Os modos de organização da atenção domiciliar no contexto da saúde ofertada nos municípios que aderiram ao Programa detectou o surgimento de um modo de organização da oferta coerente com as demandas da gestão, com os modos de organização mediados pelas necessidades dos usuários e com o perfil de saúde local(28-30).

Um estudo de inspiração genealógica objetivou problematizar a atenção domiciliar como dispositivo de segurança, proposto pelo Programa Melhor em Casa, e concluíram que a casa constitui um lugar mais seguro e melhor para o paciente, que fica próximo a sua família, sem risco de infecção hospitalar e recebendo atendimento de equipe que propiciará a tecnologia necessária(31).

O atendimento no domicílio é apresentado como melhor opção pelo conforto do lar, vínculo com a família e com a equipe, e pela superação quanto às barreiras de acesso a outros pontos da rede de atenção à saúde. $\mathrm{Na}$ perspectiva dos usuários, o cuidar em casa apresenta novas relações, as quais ampliam o acesso, a autonomia e a qualidade de vida do usuário(29,31,32).

O Parecer técnico n. ${ }^{\circ}$ 05/GEAS/GGRAS/DIPRO/2018 ${ }^{(33)}$, da Agência Nacional de Saúde, que determina a cobertura pela assistência domiciliar, estabelece a não inclusão da assistência à saúde no ambiente domiciliar (home care) entre as coberturas obrigatórias, podendo ser oferecida pelas operadoras como alternativa à internação hospitalar. Releva enfatizar que somente o médico assistente do beneficiário poderá determinar se há ou não indicação de internação domiciliar em substituição à internação hospitalar. A operadora não pode suspender uma internação hospitalar pelo simples pedido de home care. Caso a operadora não concorde em oferecer o serviço de assistência domiciliar, deverá manter o beneficiário internado até sua alta hospitalar ${ }^{(33)}$.

No presente estudo evidencia-se a necessidade de ser efetuado um número maior de estudos englobando a relação custo-benefício, a satisfação do cliente e da família, a eficácia e a efetividade desse tipo de assistência, além das vantagens e das desvantagens para um estabelecimento de saúde incorporar a atenção domiciliar como parte de seus serviços, visando ao cuidado integral e promovendo a saúde daqueles dependentes de cuidados.

\section{CONCLUSÃO}

As evidências científicas apontam a atenção domiciliar como uma ferramenta a ser utilizada na reorganização do sistema de saúde, na prevenção de agravos e na promoção da saúde.

\section{CONFLITOS DE INTERESSE}

As autoras afirmam que não houve conflitos de interesses na execução desta pesquisa.

\section{CONTRIBUIÇÕES}

Carmem Rita Sampaio de Sousa e Maria Socorro de Sousa contribuíram com a elaboração e delineamento do estudo; a aquisição, análise e interpretação dos dados; e a redação do manuscrito.

\section{FONTES DE FINANCIAMENTO}

Esta pesquisa foi financiada pela Coordenação de Aperfeiçoamento de Pessoal do Ensino Superior (Capes).

\section{REFERÊNCIAS}

1. Kerber NPC, Kirchhof ALC, Cezar-Vaz MR. Considerações sobre atenção domiciliaria e suas aproximações como mundo do trabalho na saúde. Cad Saúde Pública [Internet]. 2008 [acesso em 2019 Jan 10];24(3):488-

93. Disponível em: https://www.scielosp.org/article/csp/2008.v24n3/485-493/ 
2. Ministério da Saúde (BR). Lei $n^{\circ} 8080$, de19 setembro de 1990. Dispõe sobre as condições para a promoção, proteção e recuperação da saúde, a organização e o funcionamento dos serviços correspondentes e dá outras providências [Internet]. Brasília: Ministério da Saúde; 1990 [acesso em 2018 Set 09]. Disponível em: http://www.planalto.gov.br/ccivil_03/leis/L8080.htm.

3. Brasil. Ministério da Saúde. Resolução RDC n¹1 de 22 de janeiro de 2006. Dispõe sobre o regulamento Técnico do Funcionamento de serviços que prestam atenção domiciliar [Internet]. Diário Oficial da República Federativa do Brasil, Brasília, seção I, Supl. N.21, p. 1-2 de 30 de janeiro de 2006 [acesso em 2019 Jan 10]. Disponível em: https://atencaobasica.saude.rs.gov.br/upload/arquivos/201510/0111472320141104105323rdc-anvisa-n-11-de-26-de-janeiro-de-2006.pdf

4. Joanilho AL, Joanilho MPG. A genealogia e a História são cinzas. Rev Hist Reg [Internet]. 2008 [acesso em 2019 Jan 10];13(1);95-108. Disponível em: www.revistas.uepg.br/index.php/rhr/article/download/341/268

5. Ministério da Saúde (BR), Departamento Nacional de Saúde, Divisões de Organização Hospitalar. História e evolução dos hospitais [Internet]. $2^{\mathrm{a}}$ ed. Rio de Janeiro: Ministério da Saúde; 1965 [acesso em 2019 Jan 10]. Disponivel em: http://bvsms.saude.gov.br/bvs/publicacoes/cd04_08.pdf

6. Senado Federal (BR). Constituição da República Federativa do Brasil [Internet]. Brasília: Senado Federal; 2016 [acesso em 2018 Set 27]. Disponível em: https://www2.senado.leg.br/bdsf/bitstream/handle/id/518231/ CF88_Livro_EC91_2016.pdf

7. Ministério da Saúde (BR). Portaria $n^{\circ} 648$, de 28 de março de 2006 . Aprova a Política Nacional de Atenção Básica, estabelecendo a revisão de diretrizes e normas para a organização da Atenção Básica para o Programa Saúde da Família (PSF) e o Programa Agentes Comunitários de Saúde (PACS) [Internet]. Brasília: Ministério da Saúde; 2006 [acesso em 2019 Jan 10]. Disponível em: http://bvsms.saude.gov.br/bvs/ saudelegis/gm/2006/prt0648_28_03_2006.html

8. Ministério da Saúde (BR). Portaria $n^{\circ} 2.488$, de 21 de outubro de 2011. Aprova a Política Nacional de Atenção Básica, estabelecendo a revisão de diretrizes e normas para a organização da Atenção Básica, para a Estratégia Saúde da Família (ESF) e o Programa de Agentes Comunitários de Saúde (PACS) [Internet]. Brasília: Ministério da Saúde; 2011 [acesso em 2018 Set 09]. Disponível em http://bvsms.saude.gov.br/bvs/ saudelegis/gm/2011/prt2488_21_10_2011.html

9. Ministério da saúde (BR). Portaria 2527 de 27 de outubro de 2011. Redefine a Atenção Domiciliar no âmbito do Serviço Único de Saúde [Internet]. Brasília: Ministério da Saúde; 2011 [acesso em 2018 Set 09]. Disponível em: http://bvsms.saude.gov.br/bvs/saudelegis/gm/2011/prt2527_27_10_2011.html

10. Ministério da Saúde (BR). Portaria $n^{\circ} 963$, de 27 de maio de 2013. Redefine a Atenção Domiciliar no âmbito do Sistema Único de Saúde [Internet]. Brasília: Ministério da Saúde; 2013 [acesso em 2018 Set 09]. Disponível em: http://bvsms.saude.gov.br/bvs/saudelegis/gm/2013/prt0963_27_05_2013.html

11. Whittemore R, Knaft K. The integrative review: update methodology. J Adv Nurs [Internet]. 2005 [acesso em 2019 Jan 10];52(5):546-53. Disponível em: https://www.ncbi.nlm.nih.gov/pubmed/16268861

12. Souza MT, Silva MD, Carvalho R. Integrative review: what is it? how to do it? Einstein [Internet]. 2010 [acesso em 2019 Jan 10];8(1 Pt 1):102-6. Disponível em: http://www.scielo.br/pdf/eins/v8n1/1679-4508-eins-8-1-0102. pdf

13. Fouerwerker LCM, Merhy EE. A contribuição da atenção domiciliar para a configuração de redes substitutivas de saúde, desinstitucionalização e transformação de práticas. Rev Panam Salud Pública [Internet]. 2008 [acesso em 2019 Jan 10];24(13):180-88. Disponível em: https://scielosp.org/pdf/rpsp/2008.v24n3/180-188/pt

14. Silva KL, Sena RR, Seixas CT, Fewerwerker LCM, Merhy EE. Atenção domiciliar como mudança do modelo tecnoassistencial. Rev Saúde Pública [Internet]. 2010 [acesso em 2019 Jan 10];44(1):166-76. Disponível em: http://www.scielo.br/pdf/rsp/v44n1/18.pdf

15. Silva KL, Sena RR, Castro WS. A desospitalização em um hospital público geral de Minas Gerais: desafios e possibilidades. Rev Gaúch Enferm [Internet]. 2017 [acesso em 2019 Jan 10];38(4). Disponível em: http:// www.scielo.br/pdf/rgenf/v38n4/1983-1447-rgenf-38-04-e67762.pdf

16. Cunha JJ, Santos PND, Lepinski AG, Santos MM, Hermann AP, Lacerda MR. Gerenciando a assistência 
domiciliar. Rev Baiana Enferm [Internet]. 2014 [acesso em 2019 Jan 10];28(2);186-95. Disponível em:https:// portalseer.ufba.br/index.php/enfermagem/article/view/9864

17. Wachs LS, Nunes BP, Soares UM, Facchini LA, Thumé E. Prevalência da assistência domiciliar prestada à população idosa brasileira e fatores associados. Cad Saúde Pública [Internet]. 2016 [acesso em 2019 Jan 10];32(3). Disponível em: http://www.scielo.br/pdf/csp/v32n3/0102-311X-csp-32-03-e00048515.pdf

18. Brito MJM, Andrade AM, Caçador BS, Freitas LFC, Penna CMM. Atenção domiciliar na estruturação da rede de atenção à saúde: trilhando os caminhos da integralidade. Esc Anna Nery Rev Enferm [Internet]. 2013 [acesso em 2019 Jan 10];17(4):603-10. Disponível em: http://www.scielo.br/scielo.php?pid=S1414$81452013000400603 \&$ script=sci_abstract\&tlng=pt

19. Neves ACOJ, Castro EAB, Costa SRD. Necessidades de cuidados domiciliares de enfermagem após a alta hospitalar no contexto do SUS. Cogitare Enferm [Internet]. 2016 [acesso em 2019 Jan 10];21(4):1-9. Disponível em: https://revistas.ufpr.br/cogitare/article/view/47708

20. Pozzoli SML, Cecílio LCO. Sobre o cuidar e o ser cuidado na atenção domiciliar. Saúde Debate [Internet]. 2017 [acesso em 2019 Jan 10];41(115):1116-29. Disponível em: http://www.scielo.br/scielo.php?pid=S010311 042017000401116\&script=sci_abstract\&tIng=pt

21. Andrade AM, Brito MJM, Silva KL, Montenegro LC, Caçador BS, Freitas LFC. Organização das redes de atenção à saúde na perspectiva de profissionais da atenção domiciliar. Rev Gaúch Enferm [Internet]. 2013 [acesso em 2019 Jan 10];34(1):111-7. Disponível em: http://www.scielo.br/pdf/rgenf/v34n2/v34n2a14.pdf

22. Pires MRGM, Duarte EC, Bernarda L, Göttems D, Figueiredo NVF, Spagnol CA. Fatores associados à atenção domiciliária: subsídios à gestão do cuidado no âmbito do SUS. Rev Esc Enferm USP [Internet]. 2013 [acesso em 2019 Jan 10];47(3):648-56. Disponível em: www.scielo.br/pdf/reeusp/v47n3/0080-6234reeusp-47-3-00648.pdf

23. Franco TB, Merhy EE. Atenção domiciliar na saúde suplementar: dispositivo da reestruturação produtiva. Cienc Saúde Colet [Internet]. 2008 [acesso em 2019 Jan 10];13(5):1511-20. Disponível em: http://www.scielo. $\mathrm{br} / \mathrm{pdf} / \mathrm{csc} / \mathrm{v} 13 \mathrm{n} 5 / 16 . \mathrm{pdf}$

24. Martins AA, Franco TB, Merhy EE, Feuerwerker LCM. A produção do cuidado no Programa de Atenção Domiciliar de uma Cooperativa Médica Saúde Suplementar. Physis (Rio J) [Internet]. 2009 [acesso em 2019 Jan 10];19(2):457-74. Disponível em: http://www.scielo.br/pdf/physis/v19n2/v19n2a12.pdf

25. Silva KL, Sena RR, Rodrigues AT, Araújo FL, Belga SMMF, Duarte ED. Promoção da saúde no setor suplementar: terceirização, microrregulação e implicações no cuidado. Rev Bras Enferm [Internet]. 2015 [acesso em 2019 Jan 10];68(3):482-9. Disponível em: http://www.scielo.br/pdf/reben/v68n3/0034-7167reben-68-03-0482.pdf

26. Silva KL, Sena RR, Feuerwerker LCM, Souza CG, Souza CG, Silva PM, et al. O direito à saúde: desafios revelados na atenção domiciliar na saúde suplementar. Saude Soc São Paulo [Internet]. 2013 [acesso em 2019 Jan 10];22(3):773-84. doi:10.1590/S0104-12902013000300011

27. Meneses CS, Cuginotti AP, Cecilio LCO, Reis AAC, Andreazza R, Araújo EC. Os usuários e a transição tecnológica no setor de saúde suplementar: estudo de caso de uma operadora de plano de saúde. Ciênc Saúde Colet [Internet]. 2013 [acesso em 2019 Jan 10];18(1):57-66. Disponível em: http://www. cienciaesaudecoletiva.com.br/artigos/os-usuarios-eatransicaotecnologica-no-setor-de-saude-suplementarestudo-de-caso-de-uma-operadora-de-plano-de saude/8560?id=8560

28. Ministério da Saúde (BR). Portaria no 1208 de 18 de junho de 2013. Dispõe sobre a integração do Programa Melhor em Casa (Atenção Domiciliar no âmbito do SUS) com o Programa SOS Emergências, ambos inseridos na Rede de Atenção às Urgências [Internet]. Brasília: Ministério da Saúde; 2013 [acesso em 2018 Set 09]. Disponível em: http://bvsms.saude.gov.br/bvs/saudelegis/gm/2013/prt1208_18_06_2013.html

29. Ministério da Saúde (BR). Municípios com o melhor em casa habilitado [Internet]. Brasília: Ministério da Saúde; 2018 [acesso em 2019 Jan 10]. Disponível em: http://portalarquivos2.saude.gov.br

30. Silva KL, Silva YC, Lage EG, Paiva PA, Dias OV. Por que é melhor em casa? A percepção de usuário e cuidadores da atenção domiciliar. Cogitare Enferm [Internet]. 2017 [acesso em 2019 Jan 10];22(4):e49660. 
Disponível em: www.saude.ufpr.br/portal/revistacogitare/wp-content/.../49660-219632-1-PB.pdf

31. Oliveira SG, Kruse MHL. Melhor em casa: dispositivo de segurança. Texto \& Contexto Enferm [Internet]. 2017 [acesso em 2019 Jan 10];26(1):e2660015. Disponível em: http://www.scielo.br/pdf/tce/v26n1/pt_0104-0707tce-26-01-e2660015.pdf

32. Castro EAB, Leone DRR, Santos CM, Gonçalves FCC Neta, Gonçalves JRL, Contim D, et al. Organização da atenção domiciliar com o Programa Melhor em Casa. Rev Gaúch Enferm [Internet]. 2018 [acesso em 2019 Jan 10];39:e2016-0002. Disponível em: https://seer.ufrgs.br/RevistaGauchadeEnfermagem/article/view/79526

33. ANS (BR). Parecer Técnico Nº5/GEAS/GGRAS/DIPRO/2018: Cobertura: Assistência Domiciliar (HOME Care) [Internet]. 2018 [acesso em 2019 Jan 10]. Disponível em: http://www.ans.gov.br/images/stories/ parecer_tecnico/uploads/parecer_tecnico/_PARECER_052018_ASSISTNCIA\%20DOMICILIAR\%2\%20 HOME\%20CARE_VERSO\%20FINAL_21122017.pdf

\section{Endereço para correspondência:}

Carmem Rita Sampaio de Sousa

Universidade Federal do Ceará - UFC

Rua Papi Junior, $1223 / 5^{\circ}$ andar

Bairro: Rodolfo Teófilo

CEP: 60430-450 - Fortaleza - CE - Brasil

E-mail: carminhaneri83@gmail.com

Como citar: Sousa CRS, Sousa MS. Atenção domiciliar em saúde no brasil: visão dessa política por uma revisão integrativa. Rev Bras Promoç Saúde. 2019;32:9250. 